\title{
Thermal conductance of buckled carbon nanotubes
}

\author{
Fumio Nishimura $^{1}$, Takuma Shiga ${ }^{1}$, Shigeo Maruyama ${ }^{1}$, Kazuyuki Watanabe ${ }^{2}$, Junichiro Shiomi ${ }^{1,{ }^{*}}$ \\ ${ }^{1}$ Department of Mechanical Engineering, The University of Tokyo, 7-3-1 Hongo, Bunkyo-ku, Tokyo \\ 113-8656, Japan \\ ${ }^{2}$ Department of Physics, Tokyo University of Science, 1-3 Kagurazaka, Shinjuku-ku, Tokyo \\ 162-8601, Japan
}

\begin{abstract}
Knowledge of thermal conductance of carbon nanotubes under mechanical deformation is important to characterize the robustness of carbon nanotube heat conduction. In this study, using molecular dynamic simulations, we have calculated thermal conductance of an elastically buckled single-walled carbon nanotube. A local buckle was formed by mechanically bending a carbon nanotube at an angle of $60^{\circ}$, and thermal conductance through the buckle was calculated by a nonequilibrium molecular dynamics approach. The thermal conductance exhibits strong diameter dependence, correlated with the strain energy generated in the buckle. Despite the highly stained deformation, the thermal resistance across a buckle is similar to that of a point defect and heterotube junction, revealing a robust nature of carbon nanotube heat conduction to buckling deformation.
\end{abstract}

*shiomi@photon.t.u-tokyo.ac.jp 


\section{Introduction}

Over the last two decades, single-walled carbon nanotubes (SWNTs) have been celebrated for their unique properties due to their quasi-one-dimensional structure [1]. One of the properties that can greatly benefit from such a structure is lattice thermal conductivity. The covalently bonded graphitic structure gives rise to high phonon group velocities, and highly discretized azimuthal wavevectors limit phonon scattering channels. In addition, in an ideal setup (e.g. free standing), the edgeless structure prohibits surface phonon scattering. In such an ideal environment, SWNTs have been shown to exhibit extremely high thermal conductivity $\left(\sim 3000 \mathrm{Wm}^{-1} \mathrm{~K}^{-1}\right)[2,3]$.

While the reports of high thermal conductivity have motivated applications of carbon nanotubes as thermal interface materials [4-8] and additives in nanocomposites [9-12], in practice, one needs to consider various thermal resistances that manifest in the actual samples and environments. In this course, influence of impurities and defects on SWNT heat conduction has been widely explored [13-18]. More recently, reduction of thermal conductivity of carbon nanotube due to the contact with environment has been studied $[19,20]$. Another source of thermal resistance is the mechanical deformation. It has been shown both in experiments and numerical simulations that the heat conduction of carbon nanotube is robust against bending deformation, leading researchers to suggest their use for phonon wave guide [21, 22]. However, the knowledge is limited to this date when it comes to how the mechanical deformation with higher strain energy, namely buckling, influences the heat conduction [23].

Buckled CNTs are frequently found by transmission electron microscope (TEM) observations. Buckles are presumably caused by mechanical force that arises during sample preparation processes, such as transferring the sample onto TEM grids [24]. In addition, when growing SWNTs as a film, the friction between constituent SWNTs whose growth speed has a distribution is expected to cause mechanical forces that are sufficient to bend and even buckle the SWNTs. This should be particularly pronounced for vertically aligned SWNT (forest) films [25, 26], which has an attractive morphology for directional thermal transport applications, as all of the constituent SWNTs are required to grow at a similar speed to maintain the alignment.

The energetics of the buckling deformation due to bending has been extensively studied by using molecular dynamics (MD)[24, 27-30], and continuum models for larger scale systems [28, 29]. Step-by-step bending simulations using molecular dynamics model has reproduced the buckling structure observed with TEM, and found that buckling transition is completely reversible (elastic) up to large angles (more than $110^{\circ}$ [24]). The main focus of the reported works has been the critical curvature $\left(\kappa_{\mathrm{c}}\right)$ for buckling deformation, which was found to be proportional to the squared diameter $\left(d^{2}\right)[24,27]$ and to have minute length dependence [28]. This implies that the physics of bendinginduced buckling follows continuum elastic theory. One noticeable feature of the transition is that the release of strain energy due to the buckling formation is small, resulting in monotonous increase 
of strain energy with respect to the bending angle in the post buckling regime. At the same time, the stress is expected to concentrate in the buckled structure. These suggest that typical bending-induced buckles observed in the experiments to be under high local strain energy, which is a potential source of high thermal resistance.

In this study, to understand the impact of buckling deformation on SWNT heat conduction, we have performed MD simulations of a buckled SWNT. Buckling deformation was formed by mechanically bending an SWNT in stepwise to angle of $60^{\circ}$ (defined later). Thermal conductance through the buckle was then calculated by nonequilibrium MD method. Here, in order to isolate the effect of bending and buckling, we have constructed a system with a buckled structure held by quasi-straight SWNT leads. Correlation between the thermal conductance and the strain energy is investigated by performing the simulations for various SWNT diameters.

\section{Methodology}

We performed classical molecular dynamics (MD) simulations with the Tersoff-Brenner bond-order potential $[31,32]$ for carbon-carbon covalent bonds. In addition, the Lennard-Jones 12-6 potential $V_{\mathrm{ij}}=4 \varepsilon\left[\left(\sigma / r_{\mathrm{ij}}\right)^{12}-\left(\sigma / r_{\mathrm{ij}}\right)^{6}\right] f_{c}$ with parameters of $\varepsilon=2.4 \mathrm{meV}$ and $\sigma=3.37 \AA$ was adopted for the van der Waals interaction between (ith and $j$ th) carbon atoms. The cutoff function was applied to the Lennard-Jones potential as

$$
f_{\mathrm{c}}=\left\{\begin{array}{cc}
1 & \left(d-b / 2 \leq r_{i j}^{0} \leq d+b / 2\right) \\
0 & \text { otherwise }
\end{array}\right.
$$

where $r_{i j}^{0}$ is the equilibrium distance between $i$ th and $j$ th carbon atoms when the SWNT is straight, the $d$ is the SWNT diameter, and $b(=0.34 \mathrm{~nm})$ is the van der Waals distance. The cutoff function limits the van der Waals interaction to serve mainly as a repulsive force between upper and lower wall when a buckled structure is formed.

A buckling deformation was formed by bending a 202-unitcell-long $(\sim 50.1 \mathrm{~nm})$ SWNT [Fig. 1(a)]. The SWNT was bent by rotating the two rigid SWNT ends (85 unit cells on each end) around the origin in the opposite directions [Fig. 1(b)]. Here, the origin is the center of the initial unbent SWNT. The SWNT was bent by $1.0 \times 10^{-5}$ degree per time step $\left(2.0 \times 10^{-5} \mathrm{deg} / \mathrm{fs}\right)$ until the bending angle [Fig. 1(b)] reached $60^{\circ}$. The flexible part of the SWNT in the middle (32 unit cells, $8 \mathrm{~nm}$ long) was relaxed at every time step and formed a buckle when the local curvature exceeds the critical value [24]. During the bending, the distance between the end unit cell of the 8-nm-part and the origin was kept constant with the unit cell plane being orthogonal to the vector connecting the origin and the unit cell. This was followed by equilibration [Fig. 1(c)] and nonequilibrium MD simulations [Fig. 1(d)], where only the end unit cells of the entire SWNT were fixed and the rest of the atoms in the SWNT were allowed to move. This way, we were able to obtain a system with a local buckle held in the middle by two quasi-straight parts. Among the armchair SWNTs tested: $(10,10),(15,15),(20,20)$, 
and $(25,25)$ SWNTs, the stable buckled structures were obtained for all the SWNTs except for the $(25,25)$ SWNT, which was unstable due to the excess strain energy.

The current bending method is different from the one commonly used to study the critical bending curvature $[24,28,29]$, where the end unitcells are rotated and translated by keeping the curvature constant along the CNT (as in the beam deformation). The two methods should be similar for a small bending angle but be different for a larger one. The current bending method was adopted primarily because we were interested in the local thermal conductance at a buckle rather than the critical buckling angle. In the supercritical system $\left(\theta>\theta_{\mathrm{c}}\right)$, the bending stress on the SWNT is relaxed due to formation of a buckle, and the picture as shown in Fig. 2 is more like a buckled structure between two straight parts as often seen in the TEM observations [24]. By calculating the stress energy distribution after the buckle formation and relaxation, we have confirmed that the stress dominantly resides in the buckled part and is mostly confined within the length of $8 \mathrm{~nm}$. In addition, the current setup is particularly appropriate for thermal conductance calculations since thermal conductance of a buckle is expected to be a function of local buckling angle rather than the overall effective bending curvature. This also allows us to decouple the effect of buckling from that of SWNT-lead bending. The bending is expected to have minor influence on the CNT thermal conductivity [22], but the decoupling is convenient for phonon modal analysis where the eigen states of leads do not change from that of the straight CNT, and thus any change we see in the lattice vibrational spectra should be due to the local buckling.

After equilibrating the system at $300 \mathrm{~K}$, thermal conductance at the buckling deformation was calculated by non-equilibrium MD simulations. Temperature of both ends of SWNT colored by blue and red regions in Fig. 1(d) were maintained at $T_{\mathrm{C}}=290 \mathrm{~K}$ and $T_{\mathrm{H}}=310 \mathrm{~K}$, respectively, by using the Nose-Hoover thermostat to drive a steady thermal current $Q$ through the SWNT. The length of the temperature-controlled regions was taken to be $L_{\mathrm{ctrl}}=L / 2$, where $L$ is the length of the phonon conduction channel. The relaxation time of the Nose-Hoover thermostat was set to be $\tau=40 \mathrm{ps}$. These parameters $L_{\text {ctrl }}$ and $\tau$ were chosen to minimize the virtual thermal resistance between the channel and the thermostats [33]. The velocity Verlet algorithm was employed to integrate the classical Newton's equation of motion with the time step of $0.5 \mathrm{fs}$. The steady state thermal current was obtained by $Q=<\left(Q_{\mathrm{H}^{-}} Q_{\mathrm{C}}\right) / 2>$, where $Q_{\mathrm{H}}$ and $Q_{\mathrm{C}}$ are the thermal current into the hot and cold thermostat, respectively. Then the thermal conductance can be calculated as $K=Q /(A \Delta T)$, where $\Delta T$ is the temperature difference between arbitral locations along in the $z$ direction. Here, the cross-sectional area $A$ of an SWNT is defined using a ring of van der Waals thickness $\pi b d$.

\section{Results and discussion}


Figure 3(a) shows the total strain energy $\left(E_{s}\right)$ as a function of bending angle of armchair SWNTs with different diameters. The total stain energy is calculated by summing the difference between the potential energy of $\alpha$ th atom in $z$ th unit cell of bent $(E)$ and that of straight SWNT $\left(E^{0}\right)$ SWNTs as,

$$
E_{s}=\sum_{\alpha, z}^{N}\left(E_{\alpha, z}-E_{\alpha, z}^{0}\right)
$$

where $N$ is the number of carbon atoms. The kink in the profile denotes the buckling transition, which shows that SWNTs with smaller diameter require larger bending angle and strain energy to form a buckle. The critical curvature can be estimated from $\kappa_{\mathrm{c}}=\theta_{\mathrm{c}} / L_{\mathrm{flex}}$, where $L_{\mathrm{flex}}(=8 \mathrm{~nm})$ is the length of the flexible part during the bending simulations. The obtained critical curvature and its diameter dependence agree well with those in the previous reports $[28,29]$ despite the above mentioned difference in the bending methods. In this work, thermal conductance of buckled structures was calculated for a bending angle of $60^{\circ}$, well-above $\theta_{\mathrm{c}}$, where the strain energy exhibits nonlinear dependence on the bending angle.

While the above result shows that the total stain energy at certain bending angle is larger for larger SWNT diameter, the strain energy per atom is more appropriate measure to be correlated with thermal conductance per unit cross-sectional area $A$, where $A$ is defined to be proportional to the number of atoms in the unit cell. Hence one needs to consider the strain energy distribution that is highly inhomogeneous, as shown in Fig. 3 (b) in terms of the strain energy per unit cell along SWNT axis,

$$
E_{z}=\sum_{\alpha}^{n} E_{\alpha, z}
$$

where $n$ is the number of atoms per unit cell. As in Fig. 3 (c), the length of the strained region is better illustrated by plotting the cumulative strain energy,

$$
E_{z^{\prime}}=\sum_{z_{0}-z^{\prime} / 2}^{z_{0}+z^{\prime} / 2}
$$

where $\left(z_{0}=101\right)$ denotes the center unit cell of the 202-unit-cell-long SWNT. If we define the effective buckled region to be the region possessing 95 percent of the entire stress caused by bending and buckling, the number of unit cells in the buckle $z_{b}$ satisfies $E_{z^{\prime}=z_{b}}=0.95 E_{s}$. Then we obtain the averaged strain energy per atom $\hat{E}_{s}=E_{s} /\left(n z_{b}\right)$. As shown in Fig. 3(d), for a bending angle of $60^{\circ}, \hat{E}_{s}$ increases roughly linearly with decreasing SWNT diameter.

Figure 4 (a) shows the steady state temperature profiles of straight $(10,10),(15,15)$ and $(20,20)$ SWNTs during the NEMD simulations. The linear temperature profiles almost collapse on top of each other. By setting $\Delta T=T_{\mathrm{H}}-T_{\mathrm{C}}$, the thermal conductance of straight SWNTs was calculated to be 6.18, 7.15 and $7.18 \mathrm{GWm}^{-2} \mathrm{~K}^{-1}$ for $(10,10),(15,15)$ and $(20,20)$ SWNTs, respectively. This corresponds to thermal conductivity of around $160 \mathrm{Wm}^{-1} \mathrm{~K}^{-1}$, in agreement with the previous MD studies on heat conduction of finite-length straight SWNTs [33]. Similarly, Fig. 4(b) shows the 
temperature profile of buckled SWNTs. The temperature jumps at the buckling region decrease with increasing diameter and their values are 5.44, 5.05 and $4.03 \mathrm{~K}$ for $(10,10),(15,15)$ and $(20,20)$ SWNTs, respectively. By taking these temperature jumps as $\Delta T$, thermal resistance and conductance across the buckling deformation were obtained as shown in Fig. 5. The figure shows that the thermal resistance of a buckle increases linearly as the SWNT diameter decreases, and thus, through the diameter dependence of the strain energy discussed earlier [Fig. 3(d)], is proportional to the strain energy per atom in the buckle. The result also shows that, despite the large deformation of the buckled structure, the magnitude of the thermal resistance is moderate (tens of $\mathrm{GWm}^{-2} \mathrm{~K}^{-1}$ in terms of thermal conductance), and is similar to that of heterotube junctions and point defects.

To shed lights on the microscopic picture of thermal conductance across the buckled structure, we have performed spectral analysis of the lattice vibration. By performing equilibrium MD simulations of the straight and buckled $(10,10)$ SWNTs at $300 \mathrm{~K}$, the phonon energy spectral density was calculated by taking the power spectrum of the time history of the atomic velocity [34]. Figure 6 compares the obtained energy spectral densities of the straight and buckled $(10,10)$ SWNTs. Here, the power spectrum of the buckled SWNT was calculated at the center cell of the buckle $\left(z=z_{0}\right)$, where the influence of the strain on the spectrum is expected to be strong. Note, under the harmonic approximation and at the classical limit, the phonon energy spectral density is self-similar to local phonon density of states. Figure 6 shows that a dominant change in the spectrum by the buckling deformation occurs in the high frequency regime. The peak at around $50 \mathrm{THz}$ of the straight SWNT, which originates from the optical phonon branch of the in-plane lattice vibration, splits into two difference peaks around $47 \mathrm{THz}$ and $52 \mathrm{THz}$ when SWNT is buckled. The former peak comes from the bottom compressed part of the buckle, whose frequency is upshifted since the compression, through anharmonic potential, gives rise to the bond stiffening. Similarly, the latter peak comes from the upper stretched part of the buckle, whose frequency is downshifted due to the bond softening. On the other hand, the change in the spectrum in the low frequency regime, composed of acoustic phonons that are active heat carrier, is relatively moderate. With elastic scattering of phonons at the buckled structure in mind, the nearly constant local density of states of the active heat carriers throughout the buckled structure should help maintaining the elastic transport channels even under the buckling deformation. This is consistent with the above observation that the thermal resistance across the buckle is small despite the large deformation at the buckle, though a proof of this logic would require more detailed modal analysis.

\section{Conclusions}

By using molecular dynamics methods, thermal conductance across a bending-buckling deformation was calculated. A local buckle was formed by mechanically bending a carbon nanotube at angle of $60^{\circ}$. Nonequilibium MD simulations show that thermal resistance increases 
approximately linearly with decreasing SWNT diameter, which is suggested to be due to the correlation between thermal resistance and averaged strain energy per atom in a buckle. Despite the highly stained deformation at the buckle, the thermal resistance was found to be moderate: similar to that of a point defect and heterotube junction, revealing a robust nature of carbon nanotube heat conduction to buckling deformation.

\section{Acknowledgements}

This work was supported in part by KAKENHI 23760178, 22226006, and Global COE Program "Global Center of Excellence for Mechanical System Innovation" from the Ministry of Education, Culture, Sports, Science and Technology of Japan.

\section{References}

[1] R. Saito, G. Dresselhaus, and M. S. Dresselhaus, Physical Properties of Carbon Nanotubes, Imperial College Press, London, 1998.

[2] C. Yu, L. Shi, Z. Yao, D. Li, and A. Majumdar, Nano Lett. 5 (2005) 1842.

[3] E. Pop, D. Mann, Q. Wang, K. Goodson, and H. Dai, Nano Letters 6 (2006) 96.

[4] J. Xu and T. S. Fisher, Int. J. Heat Mass Transfer 49 (2006) 1658.

[5] X. J. Hu, A. A. Padilla, J. Xu, T. S. Fisher, and K. E. Goodson, J. Heat Transfer 128 ( 2006) 1109.

[6] B. A. Cola, J. Xu, C. Cheng, X. Xu, T. S. Fisher, and H. Hu, J. Appl. Phys. 101 (2007) 054313.

[7] M. A. Panzer, G. Zhang, D. Mann, X. Hu, E. Pop, H. Dai, and K. E. Goodson, J. Heat Transfer 130 (2008) 052401.

[8] M. A. Panzer, H. M. Duong, J. Okawa, J. Shiomi, B. L. Wardle, S. Maruyama, and K. E. Goodson, Nano Lett. 10 (2010) 2395.

[9] S. U. S. Choi, Z. G. Zhang, W. Yu, F. E. Lockwood, and E. A. Grulke, Appl. Phys. Lett. 79 (2001) 2252.

[10] M. J. Biercuk, M. C. Llaguno, M. Radosavljevic, A. J. K. Hyun, T. Johnson, and J. E. Fischer, Appl. Phys. Lett. 80 (2002) 2767.

[11] S. T. Huxtable, D. G. Cahill, S. Shenogin, L. Xue, R. Ozisik, P. Barone, M. Usrey, M. S. Strano, G. Siddons, M. Shim, and P. Keblinski, Nature Mater 2 (2003) 731.

[12] M. B. Bryning, D. E. Milkie, M. F. Islam, J. M. Kikkawa, and A. G. Yodh, Appl. Phys. Lett. 87 (2005) 161909.

[13] J. Che, T. Çagin, and W. A. Goddard III, Nanotechnology 11 (2000) 65.

[14] G. Zhang and B. Li, J. Chem Phys. 123 (2005) 114714.

[15] S. Maruyama, Y. Igarashi, T. Taniguchi, and J. Shiomi, J. Therm Sci. Tech. 1 (2006) 138.

[16] N. Kondo, T. Yamamoto, and K. Watanabe, e-J. Surf. Sci. Nanotech. 4 (2006) 239. 
[17] N. Kondo, T. Yamamoto, and K. Watanabe, Jpn. J. Appl. Phys. 45 (2006) L963.

[18] T. Yamamoto and K. Watanabe, Phys. Rev. Lett. 96 (2006) 255503.

[19] J. A. Thomas, R. M. Iutzi, and A. J. H. McGaughey, Phys. Rev. B 81 (2010) 045413.

[20] Z.-Y. Ong, E. Pop, and J. Shiomi, Phys. Rev. B (in press) (2011)

[21] C. W. Chang, D. Okawa, H. Garcia, A. Majumdar, and A. Zettl, Phys. Rev. Lett. 99 (2007) 045901 .

[22] F. Nishimura, T. Takahashi, K. Watanabe, and T. Yamamoto, APEX 2 (2009) 035003.

[23] V. Bahadur, J. Xu, Y. Liu, and T. S. Fisher, Journal of Heat Transfer 127 (2005) 664.

[24] S. Iijima, C. Brabec, A. Maiti, and J. Bernholc, J. Chem. Phys. 104 (1996) 2089.

[25] Y. Murakami, S. Chiashi, Y. Miyauchi, M. H. Hu, M. Ogura, T. Okubo, and S. Maruyama, Chem. Phys. Lett. 385 (2004) 298.

[26] K. Hata, D. N. Futaba, K. Mizuno, T. Namai, M. Yumura, and S. Iijima, Science 306 (2004) 1362.

[27] B. I. Yakobson, C. J. Brabec, and J. Bernholc, Phys. Rev. Lett. 76 (1996) 2511.

[28] G. Cao and X. Chen, Phys. Rev. B 73 (2006) 155435.

[29] Y. Sun and K. M. Liew, Comput. Methods Appl. Mech. Engrg. 197 (2008) 3001.

[30] X. Li, K. Maute, M. L. Dunn, and R. Yang, Phys. Rev. B 81 (2010) 245318.

[31] J. Tersoff, Phys. Rev. B 37 (1988) 6991.

[32] D. W. Brenner, Phys. Rev. B 42 (1990) 9458.

[33] J. Shiomi and S. Maruyama, Jpn. J. Appl. Phys. 47 (2008) 2005.

[34] J. Shiomi and S. Maruyama, Phys. Rev. B 73 (2006) 205420. 
(a) Straight SWNT

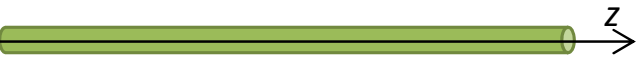

(b) Bending MD

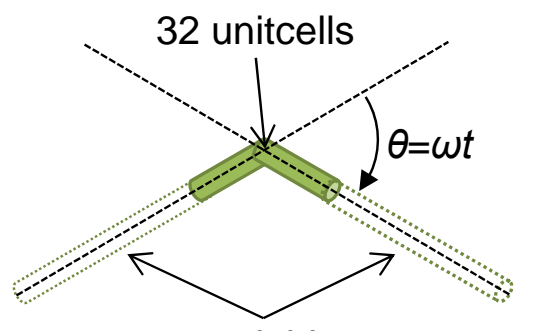

rigid

(c) Equilibrium MD@300K

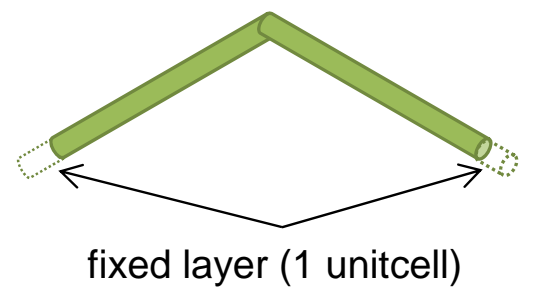

(d) Non-equilibrium MD

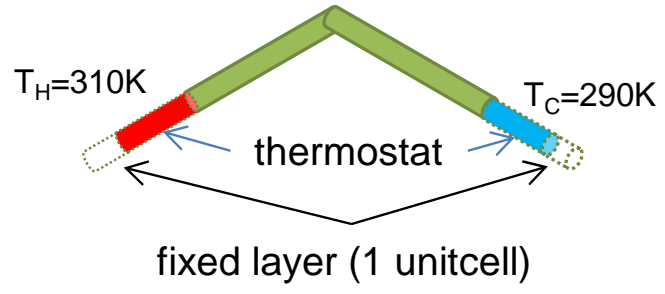

Figure 1: The procedure of molecular dynamics simulations. 


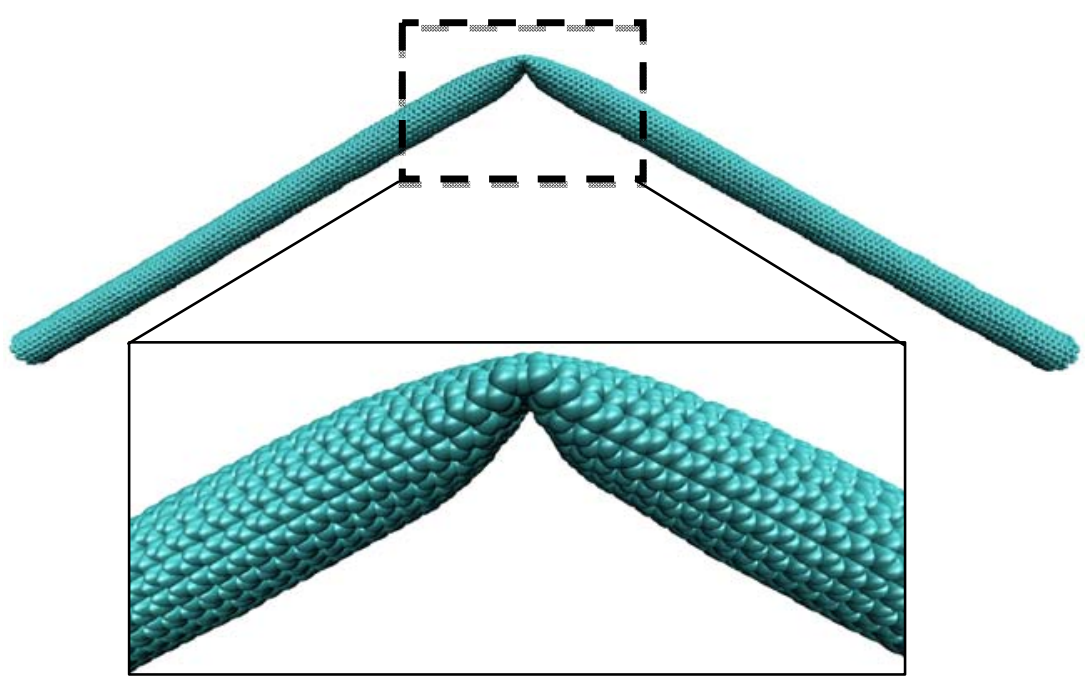

Figure 2: A typical snapshot of $(10,10)$ SWNTs after equilibration. 

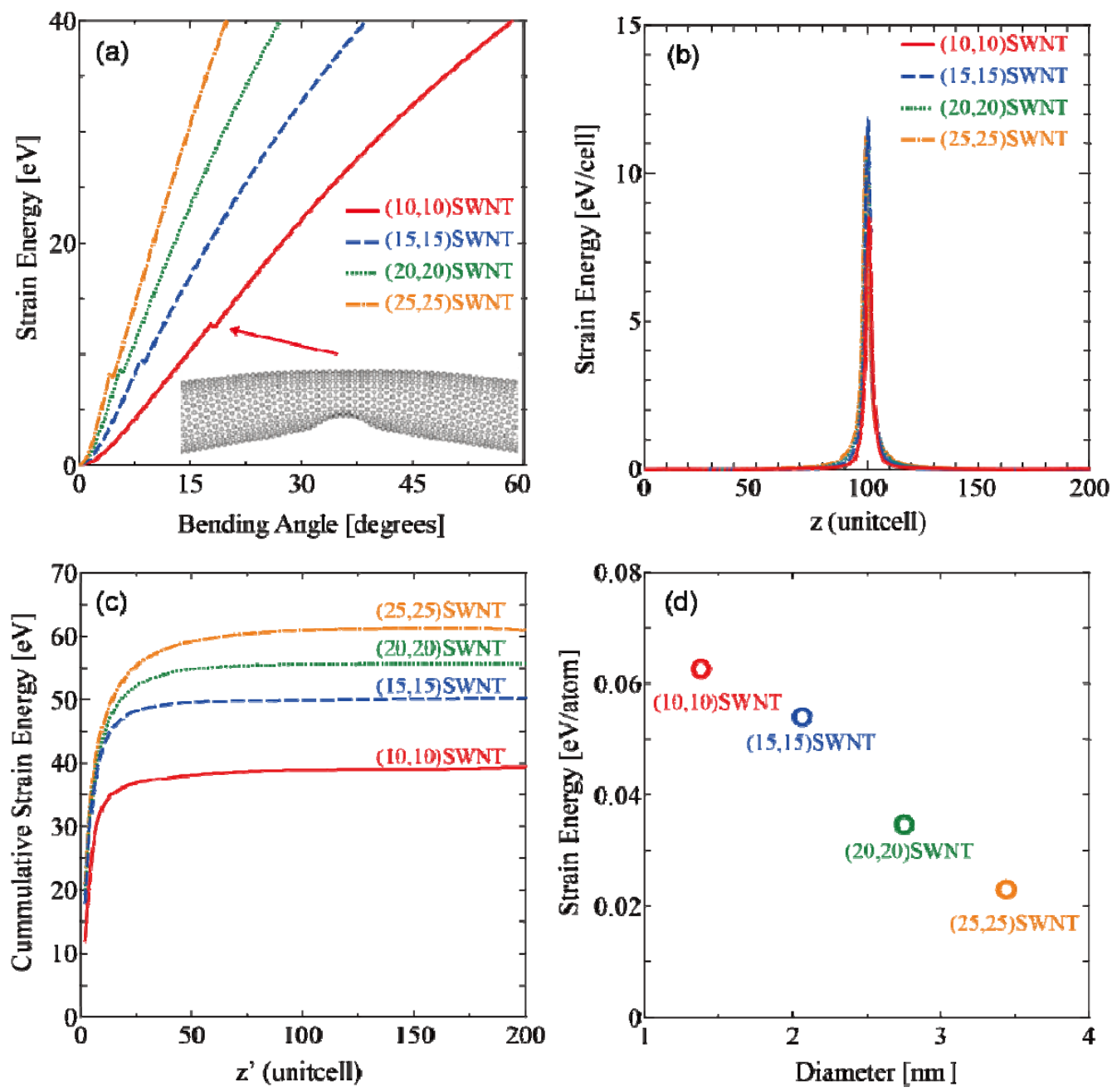

Figure 3: (a) Dependence on the bending angle $\theta$ of the total strain energy $E_{s}$ of $(10,10),(15,15)$, $(20,20),(25,25)$ SWNTs. The slight kink in the profile denotes the formation of a buckling deformation. (b) Distribution of strain energy per unit cell $E_{z}$ along the buckled SWNT. (c) The strain energy cumulated from $z^{\prime}=0$ as described in Eq. (4). (d) The diameter dependence of the effective strain energy per atom $\hat{E}_{s}$. 

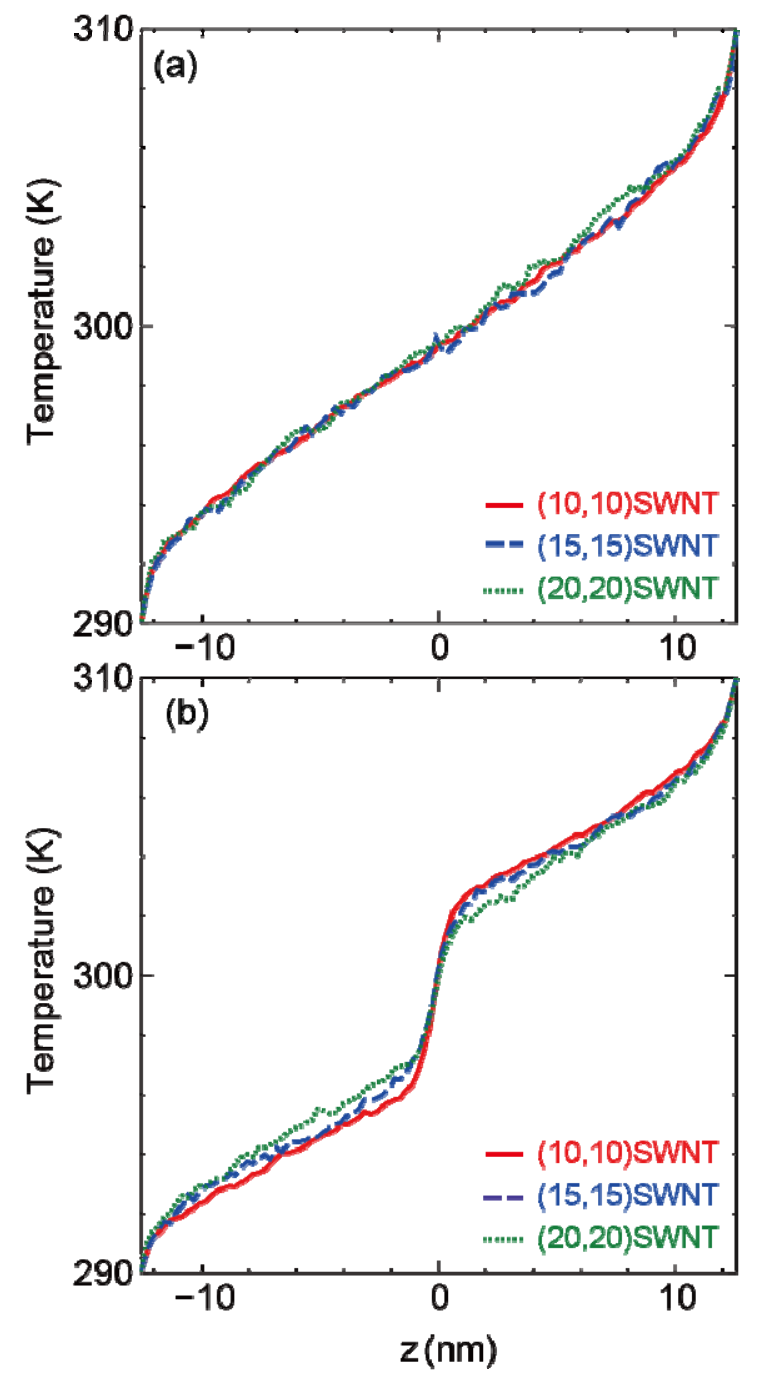

Figure 4: The temperature profiles of (a) straight and (b) buckled $(10,10),(15,15)$ and $(20,20)$ SWNTs during the nonequilibrium molecular dynamics simulations. 


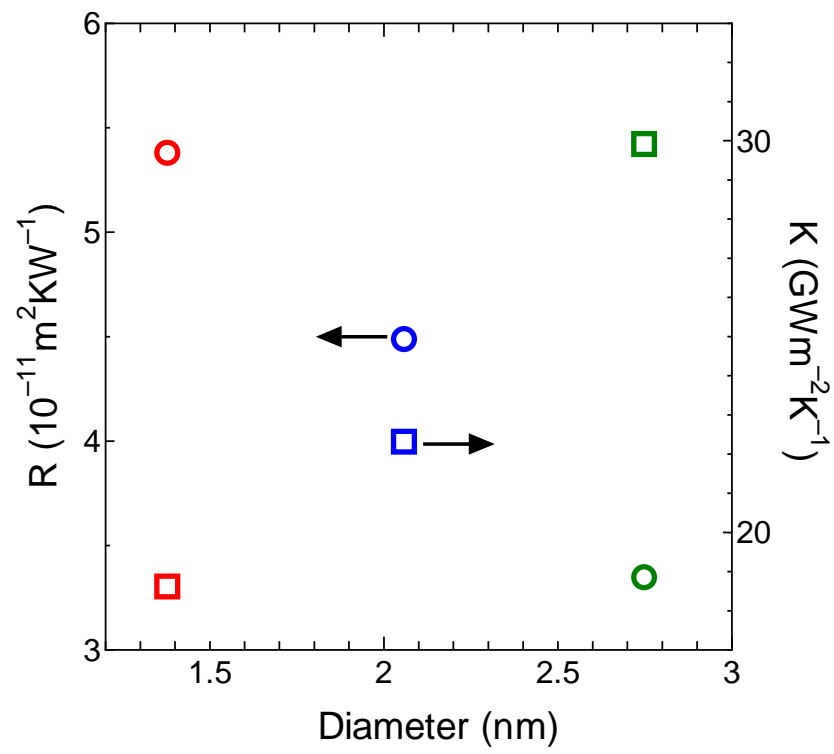

Figure 5: SWNT-diameter dependence of thermal resistance $(R=1 / K)$ and conductance $(K)$ of the buckled structure for a bending angle of $60^{\circ}$. Thermal resistance and conductance are denoted with squares and circles. 


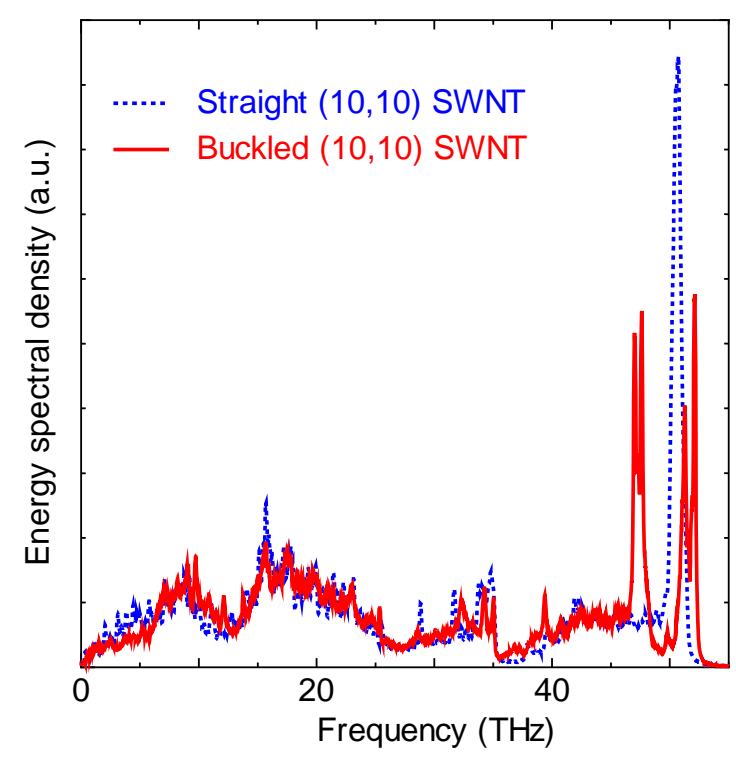

Figure 6: Energy spectral density of straight (dotted line) and buckled (solid line) $(10,10)$ SWNTs. The spectrum of the buckled SWNT is calculated at the center cell $\left(z=z_{0}\right)$. 